\title{
Coefficients of Meromorphic Bi-Bazilevic Functions
}

\author{
Jay M. Jahangiri ${ }^{1}$ and Samaneh G. Hamidi ${ }^{2}$ \\ ${ }^{1}$ Department of Mathematical Sciences, Kent State University, Burton, OH 44021-9500, USA \\ ${ }^{2}$ Institute of Mathematical Sciences, Faculty of Science, University of Malaya, 50603 Kuala Lumpur, Malaysia
}

Correspondence should be addressed to Jay M. Jahangiri; jjahangi@kent.edu

Received 19 November 2013; Accepted 6 February 2014; Published 9 March 2014

Academic Editor: Yan Xu

Copyright (C) 2014 J. M. Jahangiri and S. G. Hamidi. This is an open access article distributed under the Creative Commons Attribution License, which permits unrestricted use, distribution, and reproduction in any medium, provided the original work is properly cited.

A function is said to be bi-Bazilevic in a given domain if both the function and its inverse map are Bazilevic there. Applying the Faber polynomial expansions to the meromorphic Bazilevic functions, we obtain the general coefficient bounds for bi-Bazilevic functions. We also demonstrate the unpredictability of the behavior of early coefficients of bi-Bazilevic functions.

\section{Introduction}

Let $\Sigma$ denote the family of meromorphic functions $g$ of the form

$$
g(z)=z+b_{0}+\sum_{n=1}^{\infty} b_{n} \frac{1}{z^{n}}
$$

which are univalent in $\Delta:=\{z: 1<|z|<\infty\}$. The coefficients of $h=g^{-1}$, the inverse map of the function $g \in \Sigma$, are given by the Faber polynomial expansion:

$$
\begin{aligned}
h(w) & =g^{-1}(w)=w+B_{0}+\sum_{n=1}^{\infty} \frac{B_{n}}{w^{n}} \\
& =w-b_{0}-\sum_{n \geq 1} \frac{1}{n} K_{n+1}^{n}\left(b_{0}, b_{1}, \ldots, b_{n}\right) \frac{1}{w^{n}} ; \quad w \in \Delta,
\end{aligned}
$$

where

$$
\begin{aligned}
K_{n+1}^{n}= & n b_{0}^{n-1} b_{1}+n(n-1) b_{0}^{n-2} b_{2} \\
& +\frac{1}{2} n(n-1)(n-2) b_{0}^{n-3}\left(b_{3}+b_{1}^{2}\right) \\
& +\frac{n(n-1)(n-2)(n-3)}{3 !} b_{0}^{n-4}\left(b_{4}+3 b_{1} b_{2}\right) \\
& +\sum_{j \geq 5} b_{0}^{n-j} V_{j}
\end{aligned}
$$

and $V_{j}$ with $5 \leq j \leq n$ is a homogeneous polynomial of degree $j$ in the variables $b_{1}, b_{2}, \ldots, b_{n}$ (see [1], p. 349 or [2-4]).

For $0 \leq \alpha<1,0 \leq \beta<1, g \in \Sigma$, and $h=g^{-1}$, let $B(\alpha ; \beta)$ denote the class of bi-Bazilevic functions of order $\alpha$ and type $\beta$ (see Bazilevic [5]) if and only if

$$
\begin{gathered}
\operatorname{Re}\left(\left(\frac{z}{g(z)}\right)^{1-\beta} g^{\prime}(z)\right)>\alpha ; \quad z \in \Delta, \\
\operatorname{Re}\left(\left(\frac{w}{h(w)}\right)^{1-\beta} h^{\prime}(w)\right)>\alpha ; \quad w \in \Delta .
\end{gathered}
$$

Estimates on the coefficients of classes of meromorphic univalent functions were widely investigated in the literature. For example, Schiffer [4] obtained the estimate $\left|b_{2}\right| \leq 2 / 3$ for meromorphic univalent functions $g$ with $b_{0}=0$ and Duren ([6] or [7]) proved that if $b_{1}=b_{2}=\cdots=b_{k}=0$ for $1 \leq k<n / 2$, then $\left|b_{n}\right| \leq 2 /(n+1)$. Schober [8] considered the case where $b_{0}=0$ and obtained the estimate $\left|B_{2 n-1}\right| \leq(2 n-$ $2) ! /(n !(n-1) !)$ for the odd coefficients of the inverse function $h=g^{-1}$ subject to the restrictions $B_{2}=B_{4}=\cdots=B_{n-2}=0$ if $n$ is even or that $B_{2}=B_{4}=\cdots=B_{n-3}=0$ if $n$ is odd. Kapoor and Mishra [9] considered the inverse function $h=g^{-1}$, where $g \in B(\alpha ; 0)$, and obtained the bound $2(1-\alpha) /(n+1)$ if $((n-1) / n) \leq \alpha<1$. This restriction imposed on $\alpha$ is a very tight restriction since the class $B(\alpha ; 0)$ shrinks for large values of $n$. More recently, Hamidi et al. [10] (also see [11]) improved the coefficient estimate given by Kapoor and Mishra in [9]. 
The real difficulty arises when the bi-univalency condition is imposed on the meromorphic functions $g$ and its inverse $h=$ $g^{-1}$. The unexpected and unusual behavior of the coefficients of meromorphic functions $g$ and their inverses $h=g^{-1}$ prove the investigation of the coefficient bounds for bi-univalent functions to be very challenging. In this paper we extend the results of Kapoor and Mishra [9] and Hamidi et al. [10,11] to a larger class of meromorphic bi-univalent functions, namely, $B(\alpha ; \beta)$. We conclude our paper with an examination of the unexpected behavior of the early coefficients of meromorphic bi-Bazilevic functions which is the best estimate yet appeared in the literature.

\section{Main Results}

Applying a result of Airault [12] or [1,3] to meromorphic functions $g$ of the form (1), for real values of $p$ we can write

$$
\begin{aligned}
\left(\frac{g(z)}{z}\right)^{p} \frac{z g^{\prime}(z)}{g(z)}=1+\sum_{n=0}^{\infty} & \left(1-\frac{n+1}{p}\right) K_{n+1}^{p} \\
& \times\left(b_{0}, b_{1}, b_{2}, \ldots, b_{n}\right) \frac{1}{z^{n+1}},
\end{aligned}
$$

where

$$
\begin{aligned}
K_{n+1}^{p}= & \frac{p !}{(p-(n+1)) !(n+1) !} b_{0}^{n+1} \\
& +\frac{p !}{(p-n) !(n-1) !} b_{0}^{n-1} b_{1} \\
& +\frac{p !}{(p-n+1) !(n-2) !} b_{0}^{n-2} b_{2} \\
& +\frac{p !}{(p-n+2) !(n-3) !} b_{0}^{n-3}\left[b_{3}+\frac{p-n+2}{2} b_{1}^{2}\right] \\
& +\frac{p !}{(p-n+3) !(n-4) !} b_{0}^{n-4}\left[b_{4}+(p-n+3) b_{1} b_{2}\right] \\
& +\sum_{j \geq 5} b_{0}^{n-j} V_{j}
\end{aligned}
$$

and $V_{j}$ is a homogeneous polynomial of degree $j: 5 \leq$ $j \leq n$ in the variables $b_{1}, b_{2}, \ldots, b_{n}$. A simple calculation reveals that the first three terms of $K_{n+1}^{p}\left(b_{0}, b_{1}, b_{2}, \ldots, b_{n}\right)$ may be expressed as

$$
\begin{gathered}
\left(1-\frac{1}{p}\right) K_{1}^{p}=(p-1) b_{0}, \\
\left(1-\frac{2}{p}\right) K_{2}^{p}=(p-2)\left(\frac{p-1}{2} b_{0}^{2}+b_{1}\right), \\
\left(1-\frac{3}{p}\right) K_{3}^{p}=(p-3) \\
\times\left(\frac{(p-1)(p-2)}{6} b_{0}^{3}+(p-1) b_{0} b_{1}+b_{2}\right) .
\end{gathered}
$$

In general, for any real number $p$, an expansion of $K_{n}^{p}=$ $K_{n}^{p}\left(a_{2}, a_{3}, \ldots, a_{n}\right)$ (e.g., see [2, equation (4)] or [3]) is given by

$$
\begin{aligned}
K_{n}^{p}= & p a_{n}+\frac{p(p-1)}{2} D_{n}^{2}+\frac{p !}{(p-3) ! 3 !} D_{n}^{3}+\cdots \\
& +\frac{p !}{(p-n) ! n !} D_{n}^{n}
\end{aligned}
$$

where

$$
D_{s+m}^{m}=D_{s+m}^{m}\left(a_{1}, a_{2}, \ldots, a_{s+m}\right)=\sum_{s=1}^{\infty} \frac{m !\left(a_{1}\right)^{\mu_{1}} \cdots\left(a_{s+m}\right)^{\mu_{s+m}}}{\mu_{1} ! \cdots \mu_{s+m} !}
$$

and $a_{1}=1$. Here we note that the sum is taken over all nonnegative integers $\mu_{1}, \ldots, \mu_{s+m}$ satisfying $\mu_{1}+\mu_{2}+\cdots+$ $\mu_{s+m}=m$ and $\mu_{1}+2 \mu_{2}+\cdots+(s+m) \mu_{s+m}=s+m$. Evidently, $D_{n}^{n}\left(a_{1}, a_{2}, \ldots, a_{s+m}\right)=a_{1}^{n}$, [12]. A similar Faber polynomial expansion formula holds for the coefficients of $h$, the inverse map of $g$ (e.g., see [1, p. 349]).

The Faber polynomials introduced by Faber [13] play an important role in various areas of mathematical sciences, especially in geometric function theory (Gong [14] Chapter III and Schiffer [4]). The recent interest in the calculus of the Faber polynomials, especially when it involves $h=g^{-1}$, the inverse of $g$ (see $[1,3,12,15])$, beautifully fits our case for the meromorphic bi-univalent functions. As a result, we are able to state and prove the following

Theorem 1. For $0 \leq \alpha<1$ and $0 \leq \beta<1$ let $g \in B(\alpha ; \beta)$ and $h=g^{-1} \in B(\alpha ; \beta)$. If $b_{1}=b_{2}=\cdots=b_{n-1}=0$ for $n$ being odd or if $b_{0}=b_{1}=\cdots=b_{n-1}=0$ for $n$ being even, then

$$
\left|b_{n}\right| \leq \frac{2(1-\alpha)}{n+1-\beta}
$$

Proof. For $g \in B(\alpha ; \beta)$ and for $h=g^{-1} \in B(\alpha ; \beta)$, there exist positive real part functions $p(z)=1+\sum_{n=1}^{\infty} c_{n} z^{-n}$ and $q(w)=$ $1+\sum_{n=1}^{\infty} d_{n} w^{-n}$ in $\Delta$ so that

$$
\begin{gathered}
\left(\frac{z}{g(z)}\right)^{1-\beta} g^{\prime}(z)=\alpha+(1-\alpha) p(z)=1+(1-\alpha) \sum_{n=1}^{\infty} \frac{c_{n}}{z^{n}} \\
\left(\frac{w}{h(w)}\right)^{1-\beta} h^{\prime}(w)=\alpha+(1-\alpha) q(w)=1+(1-\alpha) \sum_{n=1}^{\infty} \frac{d_{n}}{w^{n}}
\end{gathered}
$$

Note that, according to the Caratheodory lemma (e.g., [7]), $\left|c_{n}\right| \leq 2$ and $\left|d_{n}\right| \leq 2$. 
On the other hand, by the Faber polynomial expansion, we observe that

$$
\begin{aligned}
\left(\frac{z}{g(z)}\right)^{1-\beta} g^{\prime}(z)= & \left(\frac{g(z)}{z}\right)^{\beta}\left(\frac{z g^{\prime}(z)}{g(z)}\right) \\
= & 1+\sum_{n=0}^{\infty}\left(1-\frac{n+1}{\beta}\right) \\
& \times K_{n+1}^{\beta}\left(b_{0}, b_{1}, \ldots, b_{n}\right) \frac{1}{z^{n+1}}, \\
\left(\frac{w}{h(w)}\right)^{1-\beta} h^{\prime}(w)= & \left(\frac{h(w)}{w}\right)^{\beta}\left(\frac{w h^{\prime}(w)}{h(w)}\right) \\
= & 1+\sum_{n=0}^{\infty}\left(1-\frac{n+1}{\beta}\right) \\
& \times K_{n+1}^{\beta}\left(B_{0}, B_{1}, \ldots, B_{n}\right) \frac{1}{w^{n+1}} .
\end{aligned}
$$

Comparing the corresponding coefficients of (11) and (13) we obtain

$$
(1-\alpha) c_{n+1}=\left(1-\frac{n+1}{\beta}\right) K_{n+1}^{\beta}\left(b_{0}, b_{1}, \ldots, b_{n}\right) .
$$

Similarly, from (12) and (14) we obtain

$$
(1-\alpha) d_{n+1}=\left(1-\frac{n+1}{\beta}\right) K_{n+1}^{\beta}\left(B_{0}, B_{1}, \ldots, B_{n}\right) .
$$

For the case $b_{1}=b_{2}=\cdots=b_{n-1}=0$ ( $n=$ odd), (15) and (16), respectively, upon using a simple algebraic manipulation and the fact that $B_{n}=-b_{n}$, reduce to

$$
\begin{aligned}
& \frac{(\beta-1) \cdots(\beta-(n+1))}{(n+1) !} b_{0}^{n+1} \\
& +(\beta-(n+1)) b_{n}=(1-\alpha) c_{n+1}, \\
& \frac{(\beta-1) \cdots(\beta-(n+1))}{(n+1) !} b_{0}^{n+1} \\
& -(\beta-(n+1)) b_{n}=(1-\alpha) d_{n+1} .
\end{aligned}
$$

Multiplying (18) by -1 and adding it to (17) we obtain

$$
+2(\beta-(n+1)) b_{n}=(1-\alpha)\left(c_{n+1}-d_{n+1}\right) .
$$

For the other case $b_{0}=b_{1}=b_{2}=\cdots=b_{n-1}=0$ ( $n=$ even $)$ (15) and (16), respectively, reduce to

$$
\begin{aligned}
& +(\beta-(n+1)) b_{n}=(1-\alpha) c_{n+1}, \\
& -(\beta-(n+1)) b_{n}=(1-\alpha) d_{n+1} .
\end{aligned}
$$

Solving either of (19), (20), or (21) for $b_{n}$, taking the absolute values, and applying the Caratheodory lemma we obtain $\left|b_{n}\right| \leq 2(1-\alpha) /(n+1-\beta)$.
Relaxing the coefficient restrictions imposed on Theorem 1, we experience the unpredictable behavior of the coefficients of bi-univalent functions.

Theorem 2. Let $g \in B(\alpha ; \beta), 0 \leq \alpha<1,0 \leq \beta<1$, be bi-univalent in $\Delta$. Then

(i)

$$
\left|b_{0}\right| \leq \begin{cases}\sqrt{\frac{4(1-\alpha)}{(2-\beta)(1-\beta)}}, & 0 \leq \alpha<\frac{1}{2-\beta} ; \\ \frac{2(1-\alpha)}{1-\beta}, & \frac{1}{2-\beta} \leq \alpha<1,\end{cases}
$$

(ii)

$$
\left|b_{1}\right| \leq \frac{2(1-\alpha)}{2-\beta}
$$

(iii)

$$
\begin{array}{r}
\left|b_{1}-b_{0}^{2}\right| \leq \frac{2(1-\alpha)}{2-\beta}-\frac{(1-\beta)(3-\beta)}{2}\left|b_{0}\right|^{2} \\
\text { if } \frac{5-4 \beta+\beta^{2}}{6-5 \beta+\beta^{2}} \leq \alpha<1 .
\end{array}
$$

Proof. (i) For $n \in\{0,1,2\}$ (15) yields

$$
\begin{gathered}
(1-\alpha) c_{1}=(\beta-1) b_{0}, \\
(1-\alpha) c_{2}=\frac{(\beta-1)(\beta-2)}{2 !} b_{0}^{2}+(\beta-2) b_{1}, \\
(1-\alpha) c_{3}=\frac{(\beta-1)(\beta-2)(\beta-3)}{3 !} b_{0}^{3} \\
+(\beta-3)(\beta-1) b_{0} b_{1}+(\beta-3) b_{2} .
\end{gathered}
$$

Similarly, for $n \in\{0,1,2\}$ (16) yields

$$
(1-\alpha) d_{1}=-(\beta-1) b_{0}
$$

$$
(1-\alpha) d_{2}=\frac{(\beta-1)(\beta-2)}{2 !} b_{0}^{2}-(\beta-2) b_{1} \text {, }
$$

$$
\begin{aligned}
(1-\alpha) d_{3}= & -\frac{(\beta-1)(\beta-2)(\beta-3)}{3 !} b_{0}^{3} \\
& +(\beta-3)(\beta-2) b_{0} b_{1}-(\beta-3) b_{2} .
\end{aligned}
$$

From either of the relations (25) or (28) we obtain

$$
\left|b_{0}\right| \leq \frac{2(1-\alpha)}{1-\beta}
$$

On the other hand, adding (26) and (29) yields

$$
(1-\alpha)\left(c_{2}+d_{2}\right)=(\beta-1)(\beta-2) b_{0}^{2} .
$$


Solve the above equation for $b_{0}$, take the absolute values of both sides, and apply the Caratheodory lemma to obtain

$$
\left|b_{0}\right| \leq \sqrt{\frac{(1-\alpha)\left(\left|c_{2}\right|+\left|d_{2}\right|\right)}{(1-\beta)(2-\beta)}} \leq \sqrt{\frac{4(1-\alpha)}{(1-\beta)(2-\beta)}} .
$$

Now the bounds given in Theorem 2 (i) for $\left|b_{0}\right|$ follow upon noting that

$$
\frac{2(1-\alpha)}{1-\beta} \leq \sqrt{\frac{4(1-\alpha)}{(1-\beta)(2-\beta)}} \quad \text { if } \frac{1}{2-\beta} \leq \alpha<1
$$

(ii) Multiply (29) by -1 and adding it to (26) we obtain

$$
(1-\alpha)\left(c_{2}-d_{2}\right)=2(\beta-2) b_{1}
$$

Solve the above equation for $b_{1}$, take the absolute values of both sides, and apply the Caratheodory lemma to obtain the bound $\left|b_{1}\right| \leq 2(1-\alpha) /(2-\beta)$.

(iii) From (29) we have

$$
b_{1}-b_{0}^{2}=\frac{1-\alpha}{2-\beta}\left(d_{2}-\frac{(2-\beta)(3-\beta)}{2(1-\alpha)} b_{0}^{2}\right) .
$$

Substituting for $b_{0}=((1-\alpha) /(1-\beta)) d_{1}$ and taking the absolute values of both sides we obtain

$$
\begin{aligned}
\left|b_{1}-b_{0}^{2}\right| & =\frac{1-\alpha}{2-\beta}\left|d_{2}-\frac{(1-\alpha)(2-\beta)(3-\beta)}{2(1-\beta)} d_{1}^{2}\right| \\
& =\frac{1-\alpha}{2-\beta}\left|d_{2}+\lambda d_{1}^{2}\right| .
\end{aligned}
$$

Using the fact $\left|d_{2}+\lambda d_{1}^{2}\right| \leq 2+\lambda\left|d_{1}\right|^{2}$ if $\lambda \geq-1 / 2$ which is due to the first author [16, Lemma 1] and noting that $\lambda \geq-1 / 2$ if $\alpha \geq\left(5-4 \beta+\beta^{2}\right) /\left(6-5 \beta+\beta^{2}\right)$, we obtain

$$
\left|b_{1}-b_{0}^{2}\right|=\frac{1-\alpha}{2-\beta}\left(2-\frac{(1-\alpha)(2-\beta)(3-\beta)}{2(1-\beta)}\left|d_{1}\right|^{2}\right) \text {. }
$$
obtain

$$
\left|b_{1}-b_{0}^{2}\right|=\frac{1-\alpha}{2-\beta}\left(2-\frac{(1-\beta)(2-\beta)(3-\beta)}{2(1-\alpha)}\left|b_{0}\right|^{2}\right) .
$$

Remark 3. For the special case $B(\alpha ; 0)$ we obtain the class of meromorphic bi-starlike functions. Consequently, the bound given for $\left|b_{0}\right|$ by our Theorem 2 (i) is an improvement to that given in Hamidi et al. [11, Theorem 2.i.].

\section{Conflict of Interests}

The authors declare that there is no conflict of interests regarding the publication of this paper.

\section{References}

[1] H. Airault and J. Ren, "An algebra of differential operators and generating functions on the set of univalent functions," Bulletin des Sciences Mathematiques, vol. 126, no. 5, pp. 343-367, 2002.

[2] H. Airault, "Symmetric sums associated to the factorization of Grunsky coefficients," in Conference, Groups and Symmetries, Montreal, Canada, April 2007.

[3] H. Airault and A. Bouali, "Differential calculus on the Faber polynomials," Bulletin des Sciences Mathematiques, vol. 130, no. 3, pp. 179-222, 2006.

[4] M. Schiffer, "Faber polynomials in the theory of univalent functions," Bulletin of the American Mathematical Society, vol. 54 , pp. 503-517, 1948.

[5] I. E. Bazilevic, "On a case of integrability in quadratures of the Loewner-Kufarev equation," Matematicheskii Sbornik, vol. 37, no. 79, pp. 471-476, 1955.

[6] P. L. Duren, "Coefficients of meromorphic schlicht functions," Proceedings of the American Mathematical Society, vol. 28, pp. 169-172, 1971.

[7] P. L. Duren, Univalent Functions, vol. 259 of Grundlehren der Mathematischen Wissenschaften, Springer, New York, NY, USA, 1983.

[8] G. Schober, "Coefficients of inverses of meromorphic univalent functions," Proceedings of the American Mathematical Society, vol. 67, no. 1, pp. 111-116, 1977.

[9] G. P. Kapoor and A. K. Mishra, "Coefficient estimates for inverses of starlike functions of positive order," Journal of Mathematical Analysis and Applications, vol. 329, no. 2, pp. 922934, 2007.

[10] S. G. Hamidi, S. A. Halim, and J. M. Jahangiri, "Coefficient estimates for a class of meromorphic bi-univalent functions," Comptes Rendus Mathematique, vol. 351, no. 9-10, pp. 349-352, 2013.

[11] S. G. Hamidi, S. A. Halim, and J. M. Jahangiri, "Faber polynomial coefficient estimates for meromorphic bi-starlike functions," International Journal of Mathematics and Mathematical Sciences, vol. 2013, Article ID 498159, 4 pages, 2013.

[12] H. Airault, "Remarks on Faber polynomials," International Mathematical Forum, vol. 3, no. 9-12, pp. 449-456, 2008.

[13] G. Faber, "Über polynomische Entwickelungen," Mathematische Annalen, vol. 57, no. 3, pp. 389-408, 1903.

[14] S. Gong, The Bieberbach Conjecture, vol. 12 of AMS/IP Studies in Advanced Mathematics, American Mathematical Society, Providence, RI, USA, 1999.

[15] P. G. Todorov, "On the Faber polynomials of the univalent functions of class $\Sigma$," Journal of Mathematical Analysis and Applications, vol. 162, no. 1, pp. 268-276, 1991.

[16] M. Jahangiri, "On the coefficients of powers of a class of Bazilevic functions," Indian Journal of Pure and Applied Mathematics, vol. 17, no. 9, pp. 1140-1144, 1986. 


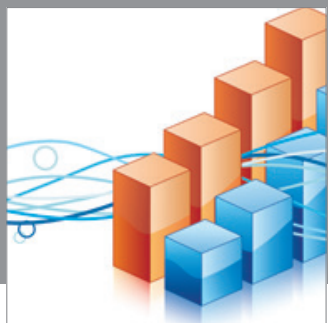

Advances in

Operations Research

mansans

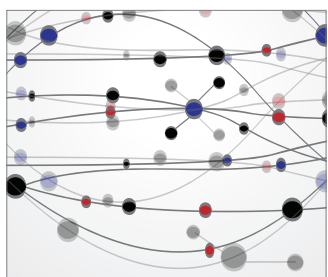

The Scientific World Journal
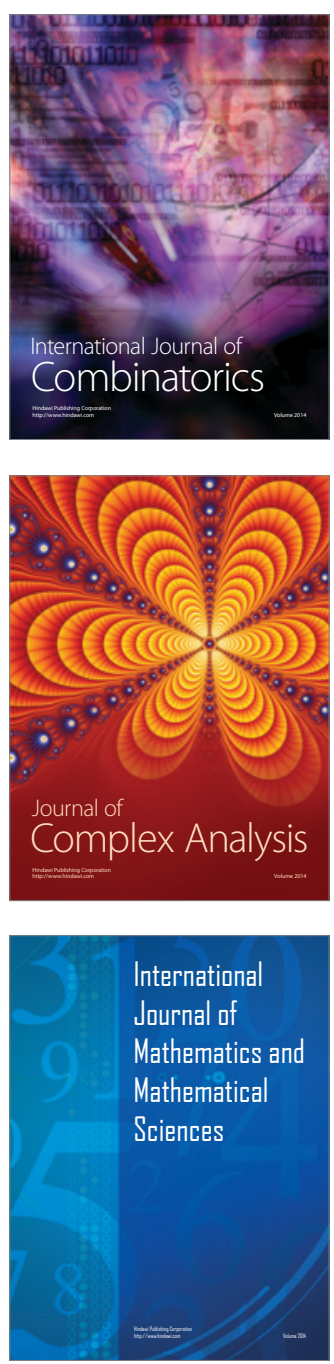
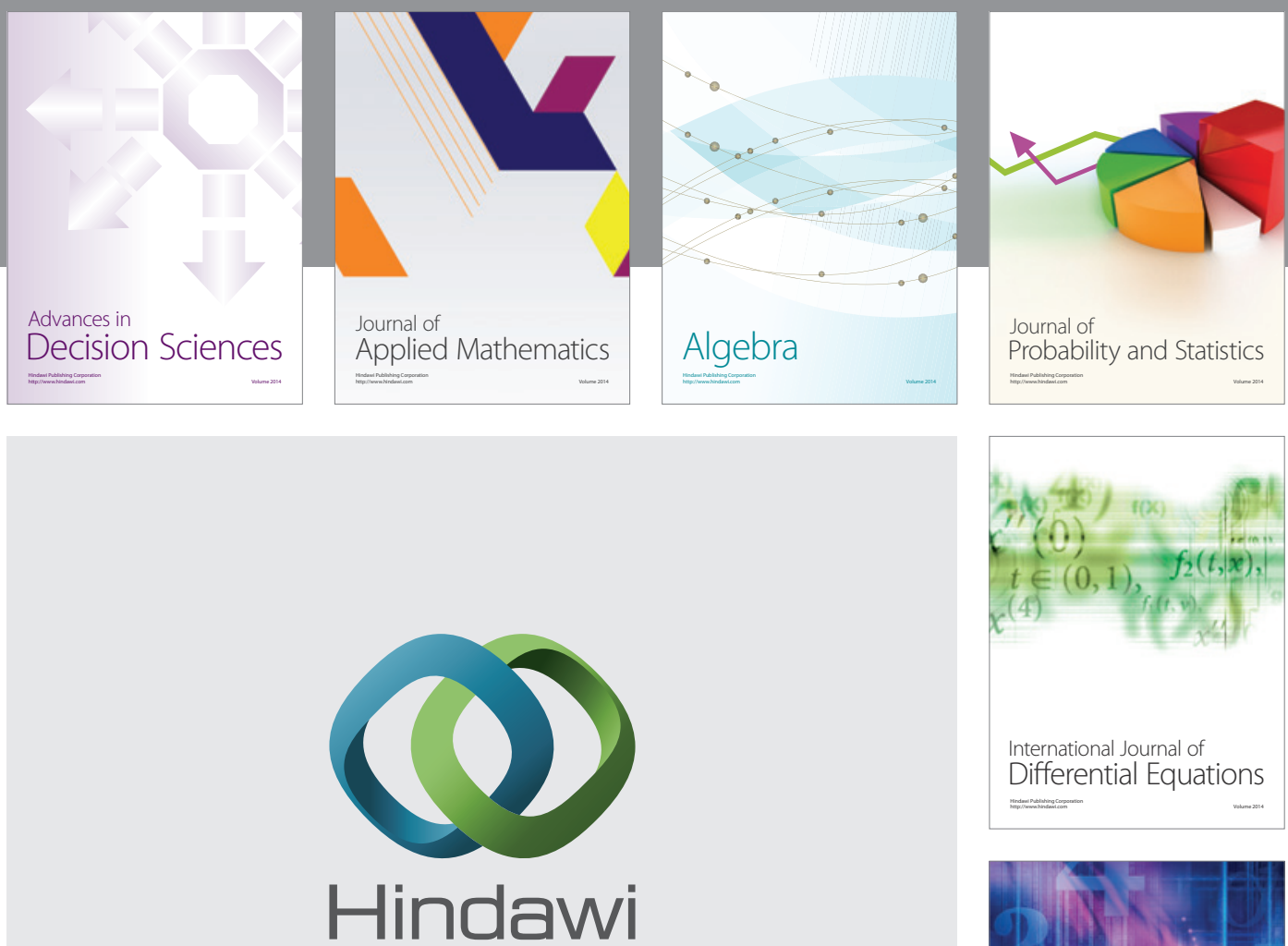

Submit your manuscripts at http://www.hindawi.com
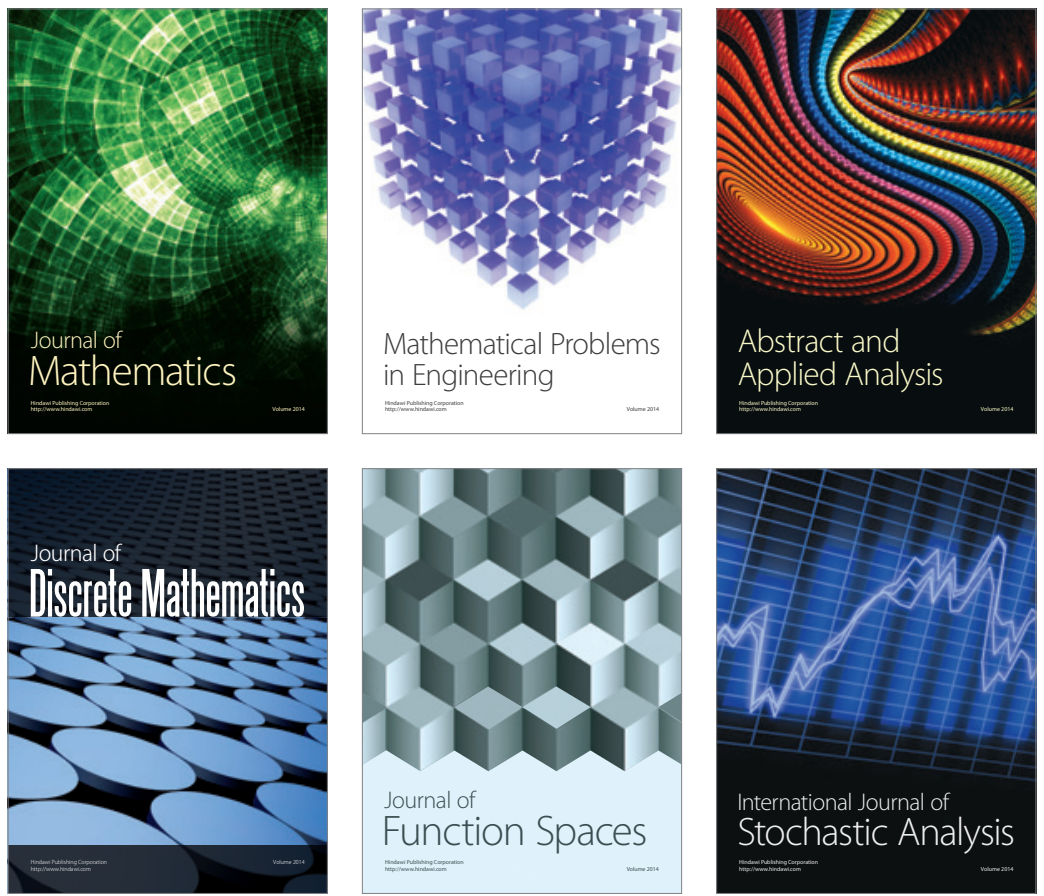

Journal of

Function Spaces

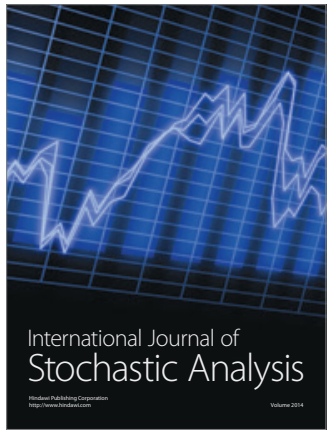

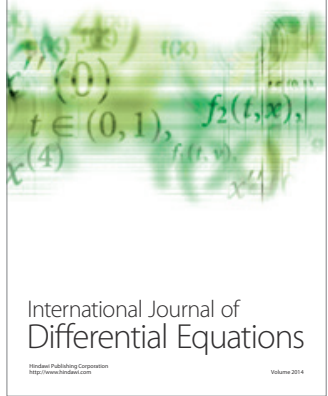
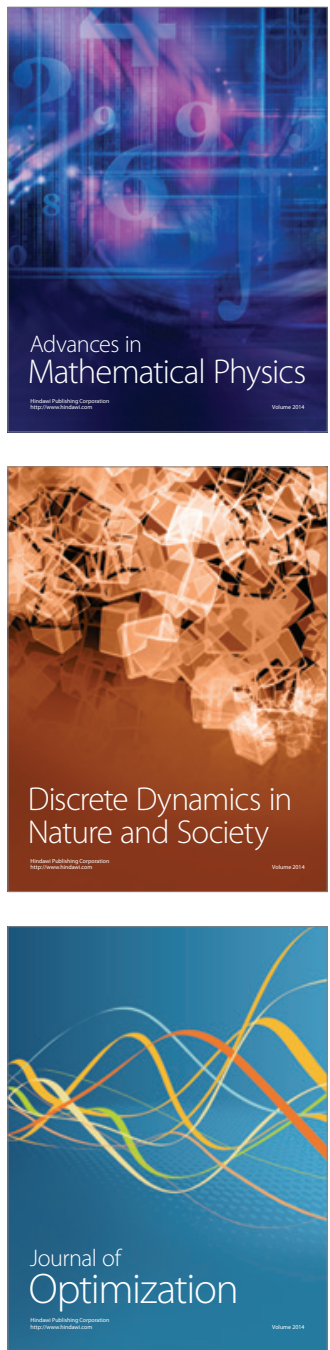